\title{
ACOMPANHAMENTO FARMACÊUTICO DE PACIENTES COM DISTÚRBIO NA GLÂNDULA DA TIREOIDE
}

\author{
PHARMACEUTICAL FOLLOW-UP OF PATIENTS WITH THYROID GLAND \\ DISORDERS
}

Jurandir Ricardo Silva dos Reis ${ }^{1}$

RESUMO: A glândula da tireoide situa-se no pescoço e é responsável pela produção de três importantes hormônios, a tri-iodotironina $\left(\mathrm{T}_{3}\right)$, a tetraiodotironina ou tiroxina $\left(\mathrm{T}_{4}\right)$ e a calcitonina, que possuem efeitos bastante relevantes no metabolismo. De uma forma geral os principais hormônios produzidos pela tireoide ( $\mathrm{T}_{3} \mathrm{e} \mathrm{T}_{4}$ ) são estimuladoras metabólicas. A produção e secreção dos hormônios $\mathrm{T}_{3} \mathrm{e}$ $\mathrm{T}_{4}$ são reguladas por um mecanismo de feedback negativo. Quando ocorre uma anomalia nesta regulação criam-se distúrbios como o hipotireoidismo, o hipertireoidismo, a doença de Graves, a tireoidite de Hashimoto, o bócio, entre outras situações clínicas.

Palavras-chave: Distúrbios da Tireoide. Atenção Farmacêutica. Tratamentos da Tireoide.

ABSTRACT: The thyroid gland is located in the neck and is responsible for the production of three important hormones, triiodothyronine $\left(\mathrm{T}_{3}\right)$, tetraiodothyronine or thyroxine $\left(\mathrm{T}_{4}\right)$ and calcitonin, which have very relevant effects on metabolism. In general, the main hormones produced by the thyroid ( $\mathrm{T}_{3}$ and $\mathrm{T}_{4}$ ) are metabolic stimulators. The production and secretion of hormones $\mathrm{T}_{3}$ and $\mathrm{T}_{4}$ are regulated by a negative feedback mechanism. When an anomaly in this regulation occurs, disorders such as hypothyroidism, hyperthyroidism, Graves' disease, Hashimoto's thyroiditis, goiter, among other clinical situations are created.

Keywords: Thyroid Disorders. Pharmaceutical Care. Thyroid Treatments.

\section{INTRODUÇÃO}

Os distúrbios da tireoide acometem a maioria da população brasileira, de maneira sintomática ou assintomática. A Tri-iodotironina $\left(\mathrm{T}_{3}\right)$ e tiroxina $\left(\mathrm{T}_{4}\right)$ são hormônios sintetizados pela glândula endócrina tireoide e o distúrbio provocado por

\footnotetext{
${ }^{1}$ Graduação em Farmácia, Nova Iguaçu-RJ,- Universidade Iguaçu, UNIG, Brasil.
} 
essas substâncias, acarretam no surgimento do Hipertireoidismo ou Hipotireoidismo (Rodrigues, Toledo, \& Nogueira, 2015), na qual podem causar inúmeras alterações no organismo (ALMEIDA et al., 2019).

Dentre as principais doenças de evolução crônica na atualidade, os distúrbios da glândula tireoide afetam diretamente as funções fisiológicas do corpo humano (FARIAS et al., 2019).

A tireoide, conhecida popularmente como glândula borboleta devido ao seu formato, é uma das maiores glândulas do sistema endócrino, na qual se encontra na região anterior do pescoço e é responsável por regular o metabolismo do corpo, bem como influenciar o funcionamento de diversos órgãos, como o coração, cérebro, fígado e os rins (RODRIGUES, TOLEDO, \& NOGUEIRA, 2015).

FIGURA r. Glândula da tiroide.

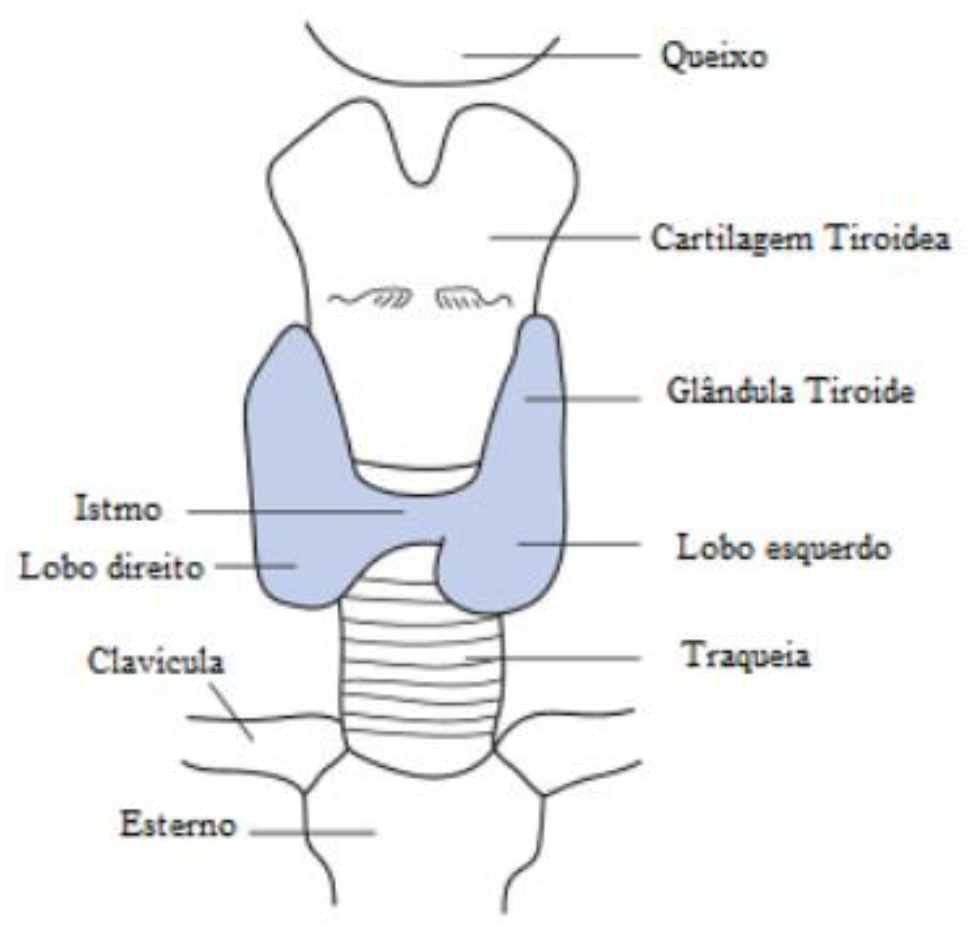

FONTE: adaptado de VANDERPUMP E TUNBRIDGE, 2008.

A glândula tireoide pode provocar diversas doenças, sendo mais comuns os nódulos, bócio, adenoma, tireoidite e câncer. Aproximadamente $5 \%$ dos indivíduos portadores do distúrbio da tireoide, desenvolveram um nódulo palpável durante alguma época da sua vida e os novos casos dessa patologia,vem aumentando de maneira gradativa anualmente (KUBO et al., 2018; MONIZ et al., 2016). 
A ação primária dos hormônios da tireoide é estimular as células ao consumo de oxigênio. Esses hormônios provocam dois distúrbios que influenciam na saúde humana. No Hipotireoidismo, a produção dos hormônios torna-se abaixo do normal e induz o metabolismo lento, na qual provoca o surgimento de alguns sintomas como bradicardia e ganho de peso (FERNANDES \& FREITAS, 2or8; PINHEIRO \& NUNES, 2019).

Enquanto que no Hipertireoidismo acontece o oposto, percebe-se a produção de hormônios acima do normal, na qual gera um aumento metabólico no organismo humano, resultando em sintomatologias como insônia, taquicardia e perda de peso (CALLEGARO \& VIANA, 2016).

Além disso, a tireoidesintetiza o hormônio combinando iodo oxidado, na forma de I3. A fusão de um átomo de iodo fornece a Monoiodotirosina ( $\mathrm{T}_{\mathrm{I}}$ ) e a segunda iodetação produz a Diiodotirosina $\left(\mathrm{T}_{2}\right)$. Com isso, duas moléculas de $\mathrm{T}_{2}$ se juntam para formar o $\mathrm{T}_{4}$, ou uma molécula de $\mathrm{T}_{1}$ e outra de $\mathrm{T}_{2}$ forma a $\mathrm{T}_{3}$ (BORTOLUZZI et al., 2016).

O hormônio que induz a maior ou menor atividade da tireoide é denominado TSH, gerado por meio da Adenohipófise ou Hipófise anterior, por um estímulo do TRH, hormônio liberador de Tirotropina. A secreção ocasionada é controlada a partir dos níveis de $\mathrm{T}_{3}$ e $\mathrm{T}_{4}$. O TSH é secretado de modo circadiano e os valores mais altos ocorrem entre duas e quatro horas da manhã, no entanto, os valores mais baixos são ocasionados entre cinco e seis horas da tarde. As variações de menor amplitude estão presentes durante o dia todo (BARROS et al., 2018).

A glândula tireoide por meio dos hormônios produzidos desenvolve funções em órgãos importantes, na regulação dos ciclos menstruais, fertilidade, peso, memória, concentração, humor e no controle emocional (CALLEGARO \& VIANA, 2016). Nesse sentido, o equilíbrio hormonal é de grande importância para o bom funcionamento da glândula tireoide, para que não haja nenhum desequilíbrio nos sistemas do corpo humano (CHENG et al., 2018), proporcionando o bem estar e uma melhor qualidade de vida.

Diante desse cenário, é necessário conhecer os distúrbios tireoidianos que acometem o indivíduo, para entender possíveis alterações e anomalias ocasionadas na 
sociedade. É importante compreender o funcionamento da glândula tireoide para diferenciar os sintomas dos distúrbios e incentivar o tratamento clínico satisfatório e eficiente.

2. OBJETIVOS:

\section{I Objetivo Geral:}

Evidenciar o papel do farmacêutico no acompanhamento a portadores dos distúrbios da tireoide.

\subsection{Objetivos Específicos:}

- Identificar as principais funções da glândula Tireoide;

- Relacionar os distúrbios da Tireoide;

- Mostrar a importância de identificar e tratar os distúrbios;

- Falar sobre a importância do farmacêutico no acompanhamento ao tratamento.

\section{METODOLOGIA:}

Essa pesquisa é qualitativa e sendo uma revisão bibliográfica utilizando um limite temporal em um período de 2015 a 2021, por meio do levantamento de dados através de periódicos, monografias, entre outros, através das bases de dados da Biblioteca Virtual em Saúde (BVS), e outras plataformas como: Literatura Latino Americana em Ciências da Saúde (Lilacs); Scientific Eletronic Library OnLine(Scielo) e Medical Literature Analysis and Retrieval System Online(Medline). De forma descritiva serão estruturados coerentemente de acordo com a proposta da pesquisa. Foram usadas as palavras chaves: Distúrbios da Tireoide; Atenção Farmacêutica, Tratamentos da Tireoide. Depois de selecionados, 
os artigos foram analisados utilizando-se os critérios de inclusão e exclusão definidos para o trabalho.

\section{JUSTIFICATIVA:}

O tema se justifica, devida a importância do farmacêutico no acompanhamento a portadores dos distúrbios da tireoide.

\section{REVISÃO DA LITERATURA:}

\section{I GLÂNDULA TIREOIDE E SUAS FUNCIONALIDADES}

A glândula tireoide regula a função de diversos e importantes órgãos como o cérebro, os rins, o coração e o fígado. Quando a tireoide sofre alguma alteração, pode diminuir a produção de hormônios causando o Hipotireoidismo ou produzir hormônios em excesso, ocasionando o Hipertireoidismo. Com isso, o volume da glândula pode aumentar, resultando na formação de bócio que surge pela falta de

iodo ou inflamação na glândula tireoide (FONSECA \& VASCONCELOS, 2org; TOMAZ et al., 2016).

A tireoide é uma glândula endócrina, localizada na região do pescoço e derivada do epitélio endodérmico da superfície mediana do assoalho faríngeo em desenvolvimento, na qual realiza liberações de secreções hormonais na corrente sanguínea (BORTOLUZZI et al., 2016).

Os hormônios tireoidianos influenciam a taxa metabólica por meio do aumento da quantidade de oxigênio utilizado nas células e na estimulação de todos os tecidos do corpo (TOMAZ et al., 2016).

Detectar possíveis alterações e as doenças provocadas pelo funcionamento irregular da tireoide possibilita um tratamento clínico satisfatório e eficiente, pois no cenário atual, a incidência do câncer de tireoide não ultrapassa 24 casos por Ioo.ooo habitantes, contudo essa taxa tem aumentado nos últimos anos, passando a ser a quarta neoplasia maligna mais frequente nas mulheres brasileiras (ROSÁRIO et al., 2013). 


\subsection{DISTÚRBIOS DA TIREÓIDE:}

\subsubsection{Gravidez e alterações na Tireoide:}

Durante a gravidez pode ocorrer alterações hormonais, que influenciem na tireoide, o hipotireoidismo na gravidez tem o maior índice decorrer, por aumento da necessidade do consumo de iodo, tendo que dobrar a quantidade ingerida. Se não houver tratamento pode ocorrer aborto, baixo peso na criança, anemia, hemorragia pós-parto e disfunção cardíaca. Caso o bebê nasça sem apresentar sintomas e sem nenhuma complicação pode ter ocorrido o hipotireoidismo congênito (de mãe para filho), por isso existe o diagnóstico no exame do pezinho (PEDROSO e TANEDA, 2014).

As gestantes que não são portadoras da doença da tireoide conseguem deixar as faixas hormonais compensadas, mas em alguns casos pode ocorrer deficiência de iodo e esse mecanismo falharem e levarem a disfunção hormonal. Para a monitoração do iodo é utilizado à avaliação da excreção de iodo urinário, conforme o valor dado como resultado conclui-se as epidemiologias (SILVA, 2017).

\subsubsection{Hipertiroidismo e Hipotireoidismo:}

O hipotireoidismo subclinico se dá pela alteração no valor sério de TSH, sendo que o $\mathrm{T}_{4}$ permanece normal e sua manifestação é assintomática, essa ausência de sintomas contribui para um agravamento dessa patologia, o hipotireoidismo é mais frequente em idosos acima de 6o anos, já que o envelhecimento causa alterações nas disposições hormonais, quando a doença manifesta sintomas em idosos, é facilmente confundida com manifestações relacionadas ao envelhecimento fisiológico, sendo assim é indispensável o diagnóstico laboratorial para que comece o tratamento, realizando o exame de dosagem do hormônio TSH principalmente em idosos e em todas as faixas etárias como profilaxia (TOMAZ et al, 2016).

Existem várias causas para o hipotireoidismo, sendo elas, autoimune, deficiência de iodo ou câncer da tireoide. O maior índice de casos da doença ocorre 
no sexo feminino com idade avançada. $O$ hipotireoidismo está associado à infertilidade, diabetes mellitus do tipo I, sendo a mais estudada do que a do tipo II porque está mais associada a fatores genéticos. $\mathrm{Na}$ depressão não existe comprovação dos locais das alterações, mas nota-se aumento dos sintomas presentes nesses pacientes como comportamento violento, demência, ansiedade e suicídio. Podendo ter duas explicações à deficiência de serotonina e noradrenalina no sistema nervoso central (PEDROSO e TANEDA, 2014).

Para diagnosticar o hipotireoidismo é necessário analisar as dosagens séricas de TSH geralmente acima de $6 \mathrm{mU} / 1, \mathrm{~T}_{4}$ livre a níveis baixos, ou uma forma subclinica TSH levemente alterado e T4l normal. $O$ tratamento é através de levotiroxina (puran $\mathrm{T}_{4}$ ) (TAVARES et al, 2016).

O hipertireoidismo ocorre por um aumento hipermetabólico que causa aumento dos níveis circulantes do hormônio $\mathrm{T}_{3}$ e $\mathrm{T}_{4}$, consequente de um aumento na função da glândula. A doença de Graves, doença autoimune, é a principal causa do hipertireoidismo, os anticorpos atacam os receptores de TSH, gerando sintomas como tremor, perda de peso, palpitações e entre outros. O hipertireoidismo pode levar a manifestações comportamentais, tais como dificuldade de aprendizagem, irritabilidade, nervosismo e distúrbios no sono, geralmente acomete pessoas com idade mais avançada e com a presença da doença por mais tempo no corpo (OLIVEIRA e MALDONADO, 2014).

O Hipertireoidismo também pode ser considerado subclinico, podendo ocorrer disfunções cardíacas devido à ação direta do $\mathrm{T}_{3}$ ou por alterações hemodinâmicas. Para tratamento é utilizado antitireoidianos, que diminuem a síntese dos hormônios e os betabloqueadores que irão controlar os batimentos cardíacos (OLIVEIRA e MALDONADO, 2014; PEDOROSO e TANEDA, 2014).

Já o tratamento para hipertireoidismo é mais complicado, em alguns casos é necessário modificar o fármaco de administração, então a primeira linha nesse caso é o metimazol, pois, normalizam rapidamente os níveis hormonais e não tem alto índice de efeitos colaterais. Os tratamentos, em grande parte dos casos, são necessários pelo fato que os hormônios da tireoide são prejudiciais na doença cardíaca (TAVARES et al, 2016). 


\subsubsection{Câncer de tireoide:}

O Câncer de tireoide é outro fator que predispõem as alterações hormonais da tireoide, geralmente é maligno, frequentemente no sexo feminino com predomínio na idade a partir de 40 anos e portadores de nódulos prévios. De acordo com o estudo no período de 2000 a 2012 para obter informações sobre o câncer da tireoide, levantouse dados sobre o aumento da taxa de mortalidade nesse período sendo um total de 6.914, assim sendo 4.702 mulheres de idade entre 60 a 79 anos sendo um reflexo do padrão dessa neoplasia, reforçando a tese que o distúrbio ocorre mais em mulheres de idade avançada, enquanto 2.212 homens contendo oscilações no mesmo período sobre os valores de óbito. Para diagnosticar o câncer é necessário o exame palpatório, em seguida, ultrassonografia e com a confirmação do nódulo é realizado a biopsia retirada do tecido para descobrir a decorrência desse nódulo sendo maligno ou benigno e as causas. Essa neoplasia esta associada a fatores biológicos, como diabetes mellitus, alcoolismo, tabagismo, dieta, atividade física, estresse e principalmente o excesso de iodo na dieta (SANTOS et al, 2016).

\subsection{FÁRMACOS UTILIZADOS NA PRÁTICA CLÍNICA}

Entre várias classes de fármacos comumente utilizados, alguns afetam a função tireoidiana. Temos os glicocorticóides, os betabloqueadores, agentes iodados, anticonvulsivantes e quimioterápicos. No entanto, o propanolol (beta-bloqueador) em excesso e a amiodarona (antiarrítmico tipo III), age inibindo a conversão do $\mathrm{T}_{4}$ em $\mathrm{T}_{3}$ e compromete a função tireoidiana significativamente. Os agentes iodados bloqueiam a iodação das tirosinas e os anticonvulsivantes, alteram o metabolismo do $\mathrm{T}_{3}$ e $\mathrm{T}_{4}$, aumentando o metabolismo hepático. O lítio inibe a síntese e liberação de $\mathrm{T}_{3}$ e $\mathrm{T}_{4}$. Os salicilatos diminuem a ligação dos hormônios tireoidianos. $\mathrm{O}$ excesso de glicocorticóides pode suprimir a secreção do TSH pela adenohipófise, além de alterar o transporte de $\mathrm{T}_{3}$ e $\mathrm{T}_{4}$ no soro (BRENTA et al., 2013).

No cenário da assistência primária à saúde do Brasil, o paciente que apresenta síndrome tireoidiana é encaminhado ao endocrinologista, após receber 
encaminhamento prévio do clínico geral na Unidade Básica de Saúde (UBS) (CORDEIRO; MARTINI, 2013).

As doenças tireoidianas são comuns e o Sistema Único de Saúde (SUS), dispõe de ensaios sensíveis e específicos ao diagnóstico e a terapêutica precisa em distúrbios com maior prevalência, cabendo ao médico generalista ter condições de identificar e fazer o manejo correto das disfunções, principalmente em síndromes tireoidianas exógenas, causadas por medicamentos (ROSS; COOPER; MULDER, 20II).

Levam-se em consideração que as patologias severas, alteram as ligações de hormônios às proteínas transportadoras onde as dosagens de TSH e o efeito dos medicamentos, apresentam um fator indispensável em testes analíticos. As interações medicamentosas são citadas envolvidas nas variações desses parâmetros. Tais influências e interferências na secreção da glândula tireóide ou no resultado de suas dosagens hormonais devem ser cuidadosamente consideradas no diagnóstico da disfunção tireoidiana (CALLEGARO; VIANA, 2012).

Diversos fármacos apresentam interação com mecanismos de ação dos hormônios tireoidianos, cujas interferências, causam confusão na interpretação dos resultados e alterações subclínicas desse perfil. Evidenciam tireoideopatias e a identificação das várias interações medicamentosas com os hormônios tireoidianos tem extrema importância no diferencial de patologias da tireoide (BRENTA et al., 2013).

Inúmeros medicamentos utilizados na prática clínica afetam a função, a ação e o metabolismo da glândula tireoide. Com a introdução de novos agentes diagnósticos e drogas, é crescente a lista de tais atividades onde alguns fármacos provocam hipotireoidismo ou hipertireoidismo. Em casos onde os processos autoimunes encontram-se envolvidos na síndrome, possivelmente, não ocorrerá o sucesso na interrupção da administração do medicamento. Alguns fármacos possuem mais de um mecanismo de ação e evidenciam anormalidades terapêuticas (CALLEGARO; VIANA, 2012). 


\subsection{ACOMPANHAMENTO FARMACOTERAPÊUTICO}

De acordo com a Organização Mundial de Saúde a principal ação da atividade farmacêutica deve ser a utilização racional dos medicamentos (OMS, 2013).

A atenção farmacêutica no contexto dos serviços farmacêuticos clínicos tem por objetivo melhorar a eficiência de um tratamento medicamentoso, esta atividade engloba atitudes, valores éticos, comportamentos, capacidade, aptidão, compromissos e obrigações a fim de prevenir doenças, promover e recuperar a saúde dos pacientes (CONSENSO BRASILEIRO DE ATENÇÃO FARMACÊUTICA, 2015).

A capacitação do farmacêutico em Farmácia Clínica surgiu para garantir o futuro da atenção farmacêutica, com novos conhecimentos o farmacêutico está preparado para desempenhar o acompanhamento farmacoterapêutico, sendo este integral e de qualidade (PEREIRA e FREITAS, 2015).

Desta forma a atenção farmacêutica consiste em especial no acompanhamento farmacoterapêutico, tendo como foco principal o paciente, buscando resultados terapêuticos satisfatórios, onde os problemas relacionados aos medicamentos serão solucionados (CIPOLLE; STRAND; MORLEY, 2015).

\section{CONCLUSÃO}

É clara a importância da função tireoidiana para os seres humanos, esta deve estar em plena conformidade de funcionamento para que se possa garantir o desempenho adequado e a homeostase do organismo. Sendo assim, orientações apropriadas de um profissional farmacêutico e de uma equipe multiprofissional em saúde, capacitados e interagindo de forma harmoniosa, vem a ser de suma importância para que o uso de medicamentos não venha a se tornar apenas uma prática paliativa ou mais um problema na vida de pacientes já fragilizados por seu estado de saúde.

A prévia anamnese farmacológica associada à triagem diagnóstica torna-se fator primordial na identificação e consequentemente na antecipação do diagnóstico das disfunções tireoidianas, reconhecendo assim, pacientes com riscos iminentes ao 
desencadeamento de tireoideopatias transitórias ou permanentes. $\mathrm{O}$ conhecimento farmacológico adquirido pelo farmacêutico na pratica clinica promove o pronto restabelecimento da saúde do paciente adequando condutas terapêuticas que trazem benefícios significativos ao bem estar dos mesmos.

Espera-se que esta abordagem metodológica possa contribuir para a melhoria na qualidade dos trabalhos na área da Atenção Farmacêutica, buscando avaliar a influência do desenvolvimento de um Acompanhamento Farmacoterapêutico em indivíduos com distúrbios da tireoide, tema esse, ainda muito incipiente no país.

\section{REFERÊNCIAS}

BARROS, ACS, Xavier, ÉM.,Reis, IS, Carvalho, PRB, Oliveira, RS, Pacheco, FK \& Queissada, DD. Farmacêutico bioquímico: uma abordagem voltada para o TSH e doenças da tireoide. Saúde e Desenvolvimento Humano. 2018.

BORTOLUZZI, TB, Yasmine CarreiraVA, Oliveira, AC, Souza, ICA, Piatto, VB \& Molina, FD. Prevalência de hipotireoidismo em pacientes com queixas de distúrbios respiratórios relacionados ao sono. Arquivos de Ciências da Saúde, 2016.

BRENTA, G; VAISMAN, M; SGARBI, JA; BERGOGLIO, LM; ANDRADA, NC; BRAVO, PP; ORLANDI, AM; GRAF, H; Em nome da Força Tarefa em Hipotiroidismo da Sociedade Latino-Americana de Tiroide (LATS). Diretrizes de prática clínica para o manejo do hipotireoidismo. Arq Bras Endocrinol Metab., $57(4): 265-299,2013$.

CALlEGARO, LF\& Viana, RO. Principais drogas que resultam em doenças da Tireoide. Revista Thêma et Scientia, 2012.

CHENG, SY, Cheng, LH, Liao, YS \& Lai, WS. Um raro tumor de laringe em paciente com carcinoma papilífero da tireoide: tumor de células granulares. Brazilian Journal of Otorhinolaryngology, 2018.

CIPOLLE, R.; STRAND, L. M.; MORLEY, P. El ejercício de la atención farmaceutica. Madrid: McGraw Hill-Interamericana; 2015. 
CONSENSO BRASILEIRO DE ATENÇÃO FARMACÊUTICA - PROPOSTA. Atenção Farmacêutica no Brasil: “Trilhando Caminhos". Brasília: Organização PanAmericana da Saúde, 2015.

CORDEIRO, EAK; MARTINI, JG. Perfil dos pacientes com câncer de tireoide submetidos à radioiodoterapia. Texto Contexto Enferm, Florianópolis, 22(4):1007IOI4, out-dez 2013.

FARIAS, KF, Leal, LR, Ribeiro, RB \& Villela, CG. Interação medicamentosa na clínica odontológica: riscos em pacientes com hipotireoidismo. Revista Brasileira de Odontologia, 2019.

FERNANDES, GQ \& Freitas, GG. Prevalência de hipotireoidismo em pacientes com diabetes mellitus tipo 2. Revista de Medicina, 2018.

FONSECA, LV \& do Vasconcelos, MJA. Repercussões de patologias tireoidianas na gestação. Revista da Faculdade de Medicina de Teresópolis. 2019.

KUBO, H, Leal, TP, Bruder, C, Bertolo, CA, Ortolani, CLF \& Takei, VLF. Disfunção da glândula tireoide e o tratamento ortodôntico: revisão integrativa da literatura. Revista Uningá, 2018.

MONIZ, C, Saraiva, C, Limbert, C, Simões, H, Carriço, M, Vasconcelos, C \& Saraiva, AM. O seguimento de lesões foliculares de significado indeterminado na citologia de nódulos da tiroideia. Revista Portuguesa de Endocrinologia, Diabetes e Metabolismo, 2016.

OLIVEIRA, R. A. Câncer diferenciado de tireoide, diagnóstico e tratamento com iodo-131. Curso de tecnologia em radiologia, Núcleo Interdisciplinar de Pesquisa 2015 .

ORGANIZACIÓN MUNDIAL DE SALUD. El papel del farmacéutico en la atención a la salud: declaración de Tokio, Genebra, 2013.

PEDROSO, B.; TANEDA, M. Análise dos casos de hipotireoidismo na região noroeste do Mato Grosso. Revista da Saúde AJES, 2014. 
PEREIRA, L. R. L.; FREITAS, O. A evolução da Atenção Farmacêutica e a perspectiva para o Brasil. Revista Brasileira de Ciências Farmacêuticas. 2015.

PINHEIRO, VP \& Nunes, CP. Manejo Terapêutico no hipotireoidismo e Gestação. Revista de Medicina de Família e Saúde Mental, 2019.

RODRIGUES, BM, Toledo, KA \& Nogueira, CR. Corpo humano: diversos sistemas interligados. Aprendendo Ciência, 2015.

ROSÁRIO, PW, Ward, LS, Carvalho, GA, Graf, H, Maciel, R, Maciel, LMZ\& Vaisman, M. Nódulo tireoidiano e câncer diferenciado de tireoide: atualização do consenso brasileiro. Arquivos Brasileiros de Endocrinologia \& Metabologia, 2013.

ROSS, DS; COOPER, DS; MULDER, JE. Laboratory assessment of thyroid function. Last update 2oli Disponível em: https://www.uptodate.com/contents/laboratory-assessmentofhyroidfunction? source $=$ search_result $\&$ search $=$ thyroxine $\&$ selected Title $=4 \% 7 E_{150}$, Acesso em: ro de setembro de 2021.

SANTOS, L. M.; et al. Evolução temporal da mortalidade por câncer de tireoide no Brasil no período de 2000 a 2012. Revista Brasileira de Análises Clínicas, pág.133-137. Aprovado ıo de março 2016.

SILVA, A.I.N. Hipotireoidismo subclínico materno durante a gestação e consequências nos filhos em idade escolar. Artigo de Revisão Bibliográfica Mestrado Integrado em Medicina, 2017.

TAVARES, M.B.; et al. A. Distúrbios da função tireoidiana induzidos pela amiodarona. Salus Journal of Health Sciences, 2016.

TOMAZ, FDD, Silva, AM, Bissoli, CF, Ferreira, RCA \& Fernandes, WS. Prevalência de Hipotireoidismo em Idosos no Município de Taubaté-SP. Rev. bras. ciênc. Saúde. 2016.

VANDERPUMP, M. P. J., Tunbridge W. M. G. (2008). The Thyroid Gland. Thyroid Diseases $4^{\circ}$ ed. Oxford, pp. 1-5. 